\title{
THE DEPENDENCE OF MASS UPON WIND
}

\author{
BRENT SMITH ${ }^{1}$
}

\begin{abstract}
As the winding number of unimodular function on $\mathbf{R}$ increases must the mass of the measure from which it arises (via the Fourier transform) increase?
\end{abstract}

We work on the real numbers, $\mathbf{R},\|\mu\|_{M}$ denotes the mass of measure $\mu$ on $\mathbf{R}$, and also we use $\|\hat{\mu}\|_{B}$ to denote the same $\|\mu\|_{M}$ where ${ }^{\wedge}$ is the standard Fourier transform. We will also use $\|f\|_{B}$ in the case that $f \neq \hat{\mu}$ for any $\mu \in M(T)$; in this case $\|f\|_{B}$ denotes $+\infty$. Let $f$ be real valued, continuous and nonlinear. H. Helson, J. P. Kahane [K, p. 87] ask if $\left\|e^{i r f}\right\|_{B}$ must exhibit at least a specific growth rate with respect to $r$. (The strong conjecture is $\left\|e^{i r f}\right\|_{B}>K \log r$ ). Now in addition let $f$ have a limit at $+\infty$. We ask a more specific question. Does there exist a function $\omega(r)$ with limit $+\infty$ at $+\infty$ and $a$ constant $K$ depending only on $\operatorname{Sup} f-\operatorname{Inf} f$ such that $\left\|e^{i r f}\right\|_{B}>K \omega(r)$ ? We motivate this question. Let $g$ be a continuous unimodular function; write $g=e^{i f}$ where $f$ is continuous. If $g$ has a finite winding number, then $f$ has limits at $\pm \infty$, say $L$ and $M$, and the winding number of $g$ is $(L-M) / 2 \pi$. Thus a yes answer to the qustion would show $\|g\|_{B}>\omega$ (winding number of $g$ ) where $\omega(r) \rightarrow \infty$ as $r \rightarrow \infty$.

THEOREM. For every $\delta>0$ there exists a function $\omega_{\delta}$ such that $\omega_{\delta}(r) \rightarrow+\infty$ as $r \rightarrow+\infty$ that satisfies: If $f$ is a real, nonconstant, continuous function on $\mathbf{R}$ that has a limit at $+\infty$. then

$$
\left\|e^{i \cdot \alpha_{r} \cdot r f}\right\|_{B}>K \omega_{\delta}(r)
$$

where $\alpha_{r} \in(1-\delta, 1]$ and where $K$ depends only on $\operatorname{Sup} f-\operatorname{Inf} f$. Furthermore, there exists a function $\omega$ such that $\omega(r) \rightarrow+\infty$ as $r \rightarrow+\infty$, that satisfies: if $f$ is in addition monotone then

$$
\left\|e^{i r f}\right\|_{B}>K \omega(r)
$$

where $K$ depends only on $\operatorname{Sup} f-\operatorname{Inf} f$.

The monotone case of the theorem has been obtained by A. M. Olevskii, [0].

ThEOREM PROOF. We use $\hat{\mu}(t)$ for $\int \bar{t} d \mu$. The proof uses the Cohen-Davenport proposition [G-McG, pp. 8-11] which we now state.

Proposition. Suppose a system of $Q^{3}+1$ functions $\left\{m_{00}\right\} \cup\left\{m_{k s}: k=\right.$ $\left.1, \ldots, Q^{2} ; s=1, \ldots, Q\right\}$ can be found that satisfy the following with respect to $a$ measure $\mu$ : Let $P_{0}=\left\{m_{00}\right\}$. $P_{k}=P_{k-1} \cup\left\{p m_{k s} \overline{m_{k t}}: p \in P_{k-1}, s<t\right\} \cup\left\{m_{k s}\right\}$.

(a) $\left\|m_{k s}\right\|_{\infty} \leq 1$

(b) $\hat{\mu}\left(m_{k s}\right) \geq 1$.

(c) $\left|\hat{\mu}\left(p m_{k s} \overline{m_{k t}}\right)\right|<e^{-Q}$ for $p \in P_{k-1}$ and $s<t$.

Then $\|\mu\|_{M}>\sqrt{Q} / 4$.

Received by the editors September 9, 1981.

1980 Mathematics Subject Classification. Primary 43A75.

${ }^{1}$ Supported by NSF Grant MCS-8102547. 
We use these notations: $\gamma_{x}(y)=e^{i x y}$. For any function $g$ and any set $S$ jump $g$ on $S=\sup _{x, y \in S}|g(x)-g(y)|$. Jump $g=$ Jump $g$ on R. Fix $Q \in \mathbf{N}$. The smallness $\epsilon=e^{-Q} / 3$ will be used. Choose $\lambda$ so that $\left|x_{i+1} / x_{i}\right|>\lambda$ for $i=1,2, \ldots$ insures $\bigcap_{i \geq 2}\left\{r: \gamma_{x_{i}}(r)\right.$ is within $\epsilon$ of $-1 ; r \in$ an interval $I$ where jump $\gamma_{x_{1}}$ on $\left.I<\epsilon\right\} \neq \emptyset$. Fix $\delta$ and choose $\lambda_{0}$ so that $2 \pi / \lambda_{0}<\delta$.

The proof idea is this. Provided only that jump $f$ is sufficiently large compared to $Q, \lambda_{0}$ we will select two systems of real numbers $\left\{x_{00}\right\} \cup\left\{x_{k s}: k=1, \ldots, Q^{2} ; s=\right.$ $1, \ldots, Q\} ;\left\{y_{00}\right\} \cup\left\{y_{k s}: k=1, \ldots, Q^{2} ; s=1, \ldots, Q\right\}$ that satisfy

(i) $\left|f\left(x_{12}\right)-f\left(y_{12}\right)\right|>\lambda_{0}$.

(ii) For $k=1,2, \ldots, Q^{2} ; s=2, \ldots, Q$

$$
\left|f\left(x_{k s}\right)-f\left(y_{k s}\right)\right|>\lambda\left|f\left(x_{k(s-1)}\right)-f\left(y_{k(s-1)}\right)\right|
$$

and for $k=2,3, \ldots, Q^{2}$

$$
\left|f\left(x_{k 2}\right)-f\left(y_{k 2}\right)\right|>\lambda\left|f\left(x_{(k-1) Q}\right)-f\left(y_{(k-1) Q}\right)\right| .
$$

(iii) Let $F_{0}=\left\{x_{00}, y_{00}\right\} . F_{k}=F_{k-1} \cup\left\{z+z_{k s}-z_{k t}: z_{k s}=x_{k s}\right.$ or $y_{k s}, z_{k t}=$ $x_{k t}$ or $\left.y_{k t}: s<t, z \in F_{k-1}\right\} \cup\left\{x_{k s}\right\} \cup\left\{y_{k s}\right\}$.

$$
\left|f\left(z+x_{k s}-x_{k t}\right)-f\left(z+x_{k s}-y_{k t}\right)\right|<\epsilon
$$

and

$$
\left|f\left(z+y_{k s}-x_{k t}\right)-f\left(z+y_{k s}-y_{k t}\right)\right|<\epsilon
$$

for $z \in F_{k-1}$ and $s<t$.

From the $x, y$ systems we construct the $m$ 's as follows. Select $r(\leq 1)$ within $2 \pi / \lambda_{0}(<\delta)$ of 1 so that $e^{i r\left(f\left(x_{k s}\right)-f\left(y_{k_{s}}\right)\right)}$ is within $\epsilon$ of -1 for all $k$ and $s=$ $2, \ldots, Q$. This is possible by (i), (ii). (This $r$ plays the role of $\alpha_{r}$ in the theorem; $\omega_{\delta}$ is determined by the number needed to dilate $f$ so that it has the required jump with respect to $\lambda_{0}, Q$ needed to find $\left\{x_{k s}, y_{k s}\right\}$.) Then let

$$
m_{k s}=\frac{1}{2}\left(e^{i r f\left(x_{k_{s}}\right)} \gamma_{x_{k_{s}}}+e^{i r f\left(y_{k_{s}}\right)} \gamma_{y_{k_{s}}}\right) \text {. }
$$

We check that this system satisfies (a), (b), (c) with respect to $\hat{\mu}=e^{i r f}$. (a), (b) present no problems. To check (c) note that since $p, m_{k s}$ have $A$-norm (= sum of the absolute values of their coefficients) $\leq 1$ we need only show: $\left|\hat{\mu}\left(\gamma_{z} \gamma_{x_{k}} \bar{m}_{k t}\right)\right|<$ $e^{-Q}$ and $\left|\hat{\mu}\left(\gamma_{z} \gamma_{y_{k}} \bar{m}_{k t}\right)\right|<e^{-Q}$ for $z \in F_{k-1}$ and $t>s$ (so $\left.t \geq 2\right)$. We do the first.

$$
\begin{aligned}
\hat{\mu}\left(\gamma_{z} \gamma_{x_{k}} \bar{m}_{k t}\right) & =\frac{1}{2}\left(e^{-i r f\left(x_{k t}\right)} \hat{\mu}\left(z+x_{k s}-x_{k t}\right)+e^{-i r f\left(y_{k t}\right)} \hat{\mu}\left(z+x_{k s}-y_{k t}\right)\right) \\
& =\frac{1}{2}\left(e^{-i r f\left(x_{k t}\right)} e^{i r f\left(z+x_{k_{s}}-x_{k t}\right)}+e^{-i r f\left(y_{k t}\right)} e^{i r f\left(z+x_{k_{s}}-y_{k t}\right)}\right) .
\end{aligned}
$$

But $e^{-i r f\left(x_{k t}\right)}$ is within $e^{-Q} / 3$ of $-e^{i r f\left(y_{k t}\right)}$ and $r \leq 1$ means $e^{i r f\left(z+x_{k \bullet}-x_{k t}\right)}$ is within $e^{-Q} / 3$ of $e^{i r f\left(z+x_{k s}-y_{k t}\right)}$ (by (iii)) and so the summands almost cancel and hence $(c)$.

We are thus left to select the $x, y$ systems. The method is to overload the jumps of $f$. Choose $w$ so that jump (of $f$ ) on $[w, \infty)<\epsilon$. The existence of $\lim _{x \rightarrow \infty} f(x)$ is needed here. Let $x_{00}=y_{00}=w$. Let $x_{11}=y_{11}=x_{00}$. Choose $w$ so that jump $\left[w, y_{11}\right]=\lambda_{0}$. Let $x_{12}>y_{12}$ be jump points. Suppose $x_{1 s}, y_{1 s}$ for $s<t$ are known with $x_{11} \geq y_{11} \geq \cdots \geq x_{1(t-1)} \geq y_{1(t-1)}$. Choose $w$ so that $\left[w, y_{1(t-1)}\right]$ has jump $\lambda^{(t-1)} \lambda_{0}$ and let $x_{1 t}>y_{1 t}$ be jump points. Note that the jump in $f$, denoted $J_{1}$, needed to form $F_{1}$ is at most $\epsilon+\lambda_{0}+\lambda_{0} \lambda+\cdots+\lambda_{0} \lambda^{(Q-1)}$. 
The rest of the argument is to show that knowledge of $J_{k-1}$ gives knowledge of $J_{k}$. Thus the required jump $J_{Q^{2}}$ can be computed recursively as a function of $\lambda_{0}, Q$.

Suppose $F_{k-1}$ is known. Card $F_{k-1}$ depends only on $Q, \lambda_{0}$; we call it $L$. Let $x_{k 1}=y_{k 1}=x_{00}$. Let $\left[w, y_{k 1}\right]$ have jump, $j_{2},=\lambda \cdot J_{k-1}\left(J_{k-1} / \epsilon\right)^{L} \cdot x_{k 2}, y_{k 2}$ will be selected from $\left[w, y_{k 1}\right]$, and hence jump on $x_{k 2}, y_{k 2}<j_{2}$. List $F_{k-1}=z_{1}, \ldots, z_{L}$. Now we overload jumps. From $\left[w, y_{k 1}\right]$ select a subset $S_{1}$ with (Lebesgue) measure of $f\left(S_{1}\right)>J_{k-1} \lambda\left(J_{k-1} / \epsilon\right)^{L-1}$ such that jump on $z_{1}+x_{k 1}-S_{1}<\epsilon$; this is possible since $\left[z_{1},+\infty\right)$ can be partitioned into $J_{k-1} / \epsilon \epsilon$-jumps. Select from $S_{1}$ a subset $S_{2}$ with measure of $f\left(S_{2}\right)>J_{k-1} \lambda\left(J_{k-1} / \epsilon\right)^{L-2}$ and jump on $z_{2}+x_{k 1}-$ $S_{2}<\epsilon$. Continuing in this manner we obtain a set $S$ with measure $f(S)>J_{k-1} \lambda$ $\left(>\left|f\left(x_{(k-1) Q}\right)-f\left(y_{(k-1) Q}\right)\right|\right)$ and jump on $z_{i}+x_{k 1}-S\left(=z_{i}+y_{k 1}-S\right)<\epsilon$ for all $i$. Choose $x_{k 2} \geq y_{k 2}$ points of $S$ that exhibit at least a $J_{k-1} \lambda$ jump. Now suppose $x_{k 1}=y_{k 1} \geq \cdots \geq x_{k(t-1)} \geq y_{k(t-1)}$ are known, and suppose $x_{k(t-1)}, y_{k(t-1)}$ were selected from $\left[w, y_{k(t-2)}\right]$ having jump $j_{t-1}$. Choose $w$ so that $\left[w, y_{k(t-1)}\right]$ has jump $j_{t}=j_{t-1} \lambda\left(J_{k-1} / \epsilon\right)^{L \cdot 2 t}$. From $\left[w, y_{k(t-1)}\right]$ we select a set $S$ with measure $f(S)>j_{t-1} \lambda$ and such that jump $z_{i}+x_{k s}-S<\epsilon$ and jump $z_{i}+y_{k s}-S<\epsilon$ for all $i$ and $s<t$. Let $x_{k t} \geq y_{k t}$ be points that exhibit at least a $j_{t-1} \cdot \lambda$ jump. Thus, $J_{k}=\epsilon+j_{2}+\cdots+j_{Q^{2}}$ works and the main theorem is proved.

Now if $f$ is in addition monotone the sets $S$ of the previous argument can be chosen to be intervals and, hence, we can choose points in $S$ having jump exactly $\pi$.

We thank Professors H. Helson and T. Ramsey for their conversations on this problem.

\section{REFERENCES}

[G-McG] C. Graham and O. C. McGehee, Essays in commutative harmonic analysis, Springer-Verlag, Berlin and New York, 1979.

[K] J. P. Kahane, Series de Fourier absolument convergentes, Springer-Verlag, Berlin and New York, 1970.

[O] A. M. Olevskii, Dokl. Akad. Nauk SSSR 256 (1981), 284-290; English transl., Soviet Math. Dokl. 23 (1981), 76-79.

Department of Mathematics, California institute of Technology, PasaDENA, CALIFORNIA 91125 